\title{
REVERSIBLE ORDER-DISORDER TRANSITION IN ETTRINGITE-METAETTRINGITE CONVERSION?
}

\author{
TULIO HONORIO ${ }^{1}$, SIRINE AL DANDACHLI ${ }^{2}$, AND ALEXANDRA BOURDOT ${ }^{3}$ \\ ${ }^{1}$ Université Paris-Saclay, ENS Paris-Saclay, CNRS, LMT - Laboratoire de Mécanique et Technologie \\ Postal Address: 91190, Gif-sur-Yvette, France \\ E-mail address: tulio.honorio-de-faria@ens-paris-saclay.fr \\ ${ }^{2}$ Université Paris-Saclay, ENS Paris-Saclay, CNRS, LMT - Laboratoire de Mécanique et Technologie \\ Postal Address: 91190, Gif-sur-Yvette, France \\ E-mail address: dandachlicirine@gmail.com \\ ${ }^{3}$ Université Paris-Saclay, ENS Paris-Saclay, CNRS, LMT - Laboratoire de Mécanique et Technologie \\ Postal Address: 91190, Gif-sur-Yvette, France \\ E-mail address: alexandra.bourdot@ens-paris-saclay.fr
}

Key words: Chemo-mechanics, Molecular Simulations, AF-phases, Osmotic ensemble, Sorption-induced deformations.

\begin{abstract}
The stability of ettringite under sorption and the conversion into metaettringite are studied using hybrid Grand Canonical Monte Carlo (GCMC) and (classical) Molecular Dynamics (MD) simulations sampling the osmotic ensemble. The desorption branch obtained from simulation and the associated volume changes are in agreement with the experimental evidence. We pay special attention to the structural changes at low RH, which is associated with metaettringite conversion, which is recognized as a disordered polymorph of ettringite with an unknown structure to date. We show that the conversion of ettringite into metaettringite is associated with an increase in entropy. The adsorption branch obtained from simulations is reversible in the ettringite domain in agreement with experiments. The reversibility in the conversion of metaettringite into ettringite, which is observed experimentally, is not captured by the simulation approach adopted. The large deformations associated with ettringite desorption make it difficult to capture reversibility with a direct sampling of the osmotic ensemble. Further, we discuss the role of hydrogen bonds on the hysteresis observed in sorption cycles in ettringite.
\end{abstract}

\section{INTRODUCTION}

Ettringite $\left(3 \mathrm{CaO} \cdot \mathrm{Al}_{2} \mathrm{O}_{3} \cdot 3 \mathrm{CaSO}_{4} \cdot n \mathrm{H}_{2} \mathrm{O}\right.$, with $\left.n=24-27\right)$ is one of the main phases formed in the hydration of ordinary and alternative cements, play a significant role in the mechanical and durability performance of cement composites. On one hand, this phase is associated with durability problems such as delayed ettringite formation. On the other hand, ettringite contributes to the immobilization of pollutants in concrete. Further, ettringite has been studied as a material for thermal energy storage (TES) due to its low cost, high energy storage density and low temperature of application $[1,2]$.

Fundamental understanding of the physical origins of the thermo-hydro-mechanical response of ettrin- 
gite is critical for the development of ettringite-based materials for TES and the comprehension of the durability problems related to delayed ettringite formation. The physical phenomena that govern the behavior of this mineral, especially with respect to hysteresis, volume stability, and reversibility of adsorption-desorption cycles are still poorly understood. At low relative humidity $(\mathrm{RH})$ the formation of metaettringite, a disordered polymorph of ettringite, is reported [4]. Atomic-level detail on the structure of metaettringite and physical phenomenon occurring in ettringite-metaettringite conversion may help in understanding the reversibility of the processes key in the utilization of ettringite for TES.

In this work, the stability of ettringite under sorption and the conversion into metaettringite at low RH are studied using hybrid Grand Canonical Monte Carlo (GCMC) and (classical) Molecular Dynamics (MD) simulations. The empirical force field AFFF developed to molecular model of AF-phases in cement systems is deployed [6]. GCMC-MD simulation allows probing the osmotic ensemble so that the water content and relaxed structures are obtained as a function of an imposed RH [7]. We show that the conversion of ettringite into metaettringite is associated with an increase in entropy. The adsorption branch obtained from simulations is reversible in the ettringite domain in agreement with experiments. The reversibility in the conversion of metaettringite into ettringite, which is observed experimentally, is not captured by the simulation approach adopted. The large deformations associated with ettringite desorption make it difficult to capture reversibility with a direct sampling of the osmotic ensemble. Further, we discuss the role of hydrogen bonds on the hysteresis observed in sorption cycles in ettringite.

\section{MATERIALS AND METHODS}
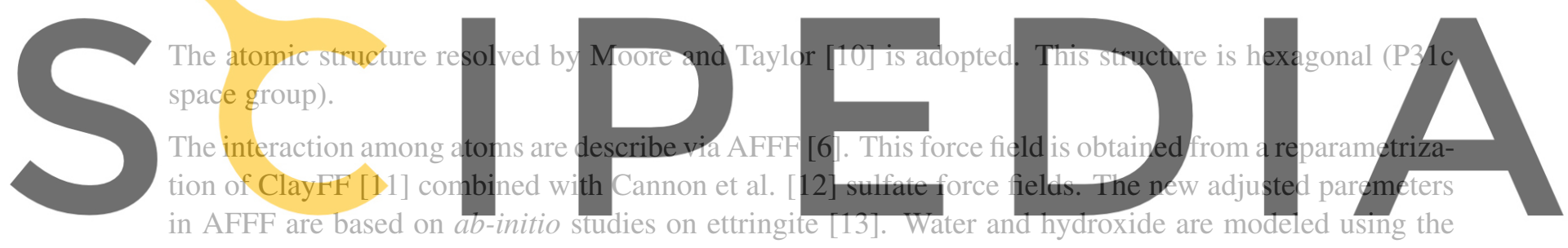
extended single-point charge (SPC/E) water model [14].

Register for free at https//www.scipedia.com to download the version without the watermark With AFFF the total energy of the system is modeled by a sum of the contributions from the short-range interactions (van der Waals forces), the electrostatic (Coulombic) interactions, and the 2- and 3-body interactions (bond and angle, respectively):

$$
U^{T o t}=U^{V d W}+U^{\text {Coul }}+U^{\text {Bond }}+U^{\text {Angle }}
$$

The nonbond interactions are described by the Lennard-Jones (12-6) potential :

$$
U^{V d W}=\sum_{i \neq j} 4 \varepsilon_{L J}\left[\left(\frac{\sigma_{L J}}{r_{i j}}\right)^{12}-\left(\frac{\sigma_{L J}}{r_{i j}}\right)^{6}\right]
$$

where $r_{i j}$ is the distance between the particles $i$ and $j$; $\varepsilon_{L J}$ and $\sigma_{L J}$ are empirical parameters. Here, Lennard-Jones parameters are defined only for the same atom type; the Lorentz-Berthelot mixing rule is employed for two dissimilar nonbond atoms. The electrostatic interactions are described by the Coulomb potential: 
Table 1: AFFF: Nonbond parameters and partial charges. The values with ${ }^{*}$ are a reparametrization proposed in AFFF.

\begin{tabular}{c|c|c|c|c}
\hline Species and Symbol & partial charge [e] & $\varepsilon_{L J}[\mathrm{~kJ} / \mathrm{mol}]$ & $\sigma_{L J}[\AA]$ & Based on \\
\hline water hydrogen, $\mathrm{H}_{w}$ & 0.4238 & - & - & $\mathrm{SPC} / \mathrm{E}[14]$ \\
water oxygen, $\mathrm{O}_{w}$ & -0.8476 & 0.650 & 3.166 & \\
\hline hydroxyl hydrogen, $\mathrm{H}_{H}$ & 0.4238 & - & - & $\mathrm{SPC} / \mathrm{E}[14]$ \\
hydroxyl oxygen, $\mathrm{O}_{H}$ & $-0.8988^{*}$ & 0.650 & 3.166 & \\
\hline sulfate oxygen, $\mathrm{O}_{S}$ & -1.0 & 0.837 & 3.15 & Cannon et al. [12] \\
sulfur, $\mathrm{S}$ & 2.0 & 1.046 & $0.10^{*}$ & \\
\hline aluminium, $\mathrm{Al}$ & $1.05^{*}$ & 0.4184 & 4.27 & ClayFF [11] \\
calcium, $\mathrm{Ca}$ & $1.6^{*}$ & 0.4184 & 5.56 & \\
\hline
\end{tabular}

Table 2: AFFF: Bonded parameters. The values with * are a reparametrization proposed in AFFF.
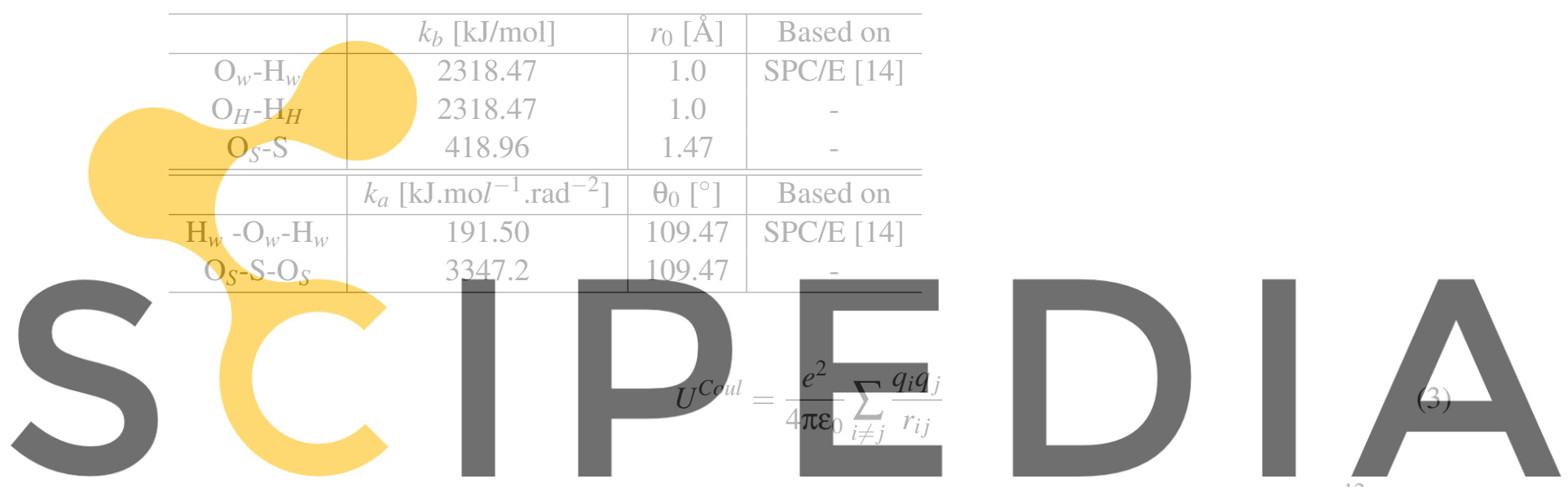

where $q_{i}$ is the partial charge of a particle $i, e$ is the elementary charge, and $\varepsilon_{0}=8.85419 \times 10^{-12} \mathrm{~F} / \mathrm{m}$ is

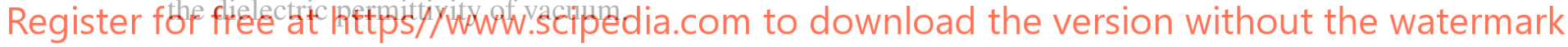

Bond (2-body) and angle (3-body) interactions are described by harmonic potentials:

$$
U^{\text {Bond }}\left(r_{i j}\right)=k_{b}\left(r_{i j}-r_{0}\right)^{2} ; U^{\text {Angle }}\left(\theta_{i j k}\right)=k_{a}\left(\theta_{i j k}-\theta_{0}\right)^{2}
$$

where $k_{b}$ and $k_{a}$ are the rigidity of the bond and angle, respectively; $r_{0}$ and $\theta_{0}$ are the equilibrium distance and angle, respectively.

Table 1 and 2 show, respectively, the nonbond and partial charges, and bond and angle parameters as defined in AFFF.

Hybrid GCMC-N $\sigma$ T simulations are carried out to simulate systems under controlled stress and RH. During the GCMC stage, water molecules may exchange with an infinite water reservoir at imposed chemical potential and temperature. For temperatures closer to ambient temperatures, an ideal gas behavior for water can be assumed since the temperature is well below the critical point for water. The chemical potential of water can be computed from the RH to be imposed using $\mu-\mu_{0} \approx k_{B} T \ln (R H)$, where $k_{B}$ is the Boltzmann constant, and $T$ is the temperature. A stress $\sigma$ in the N $\sigma \mathrm{T}$ stage, and the six components of 

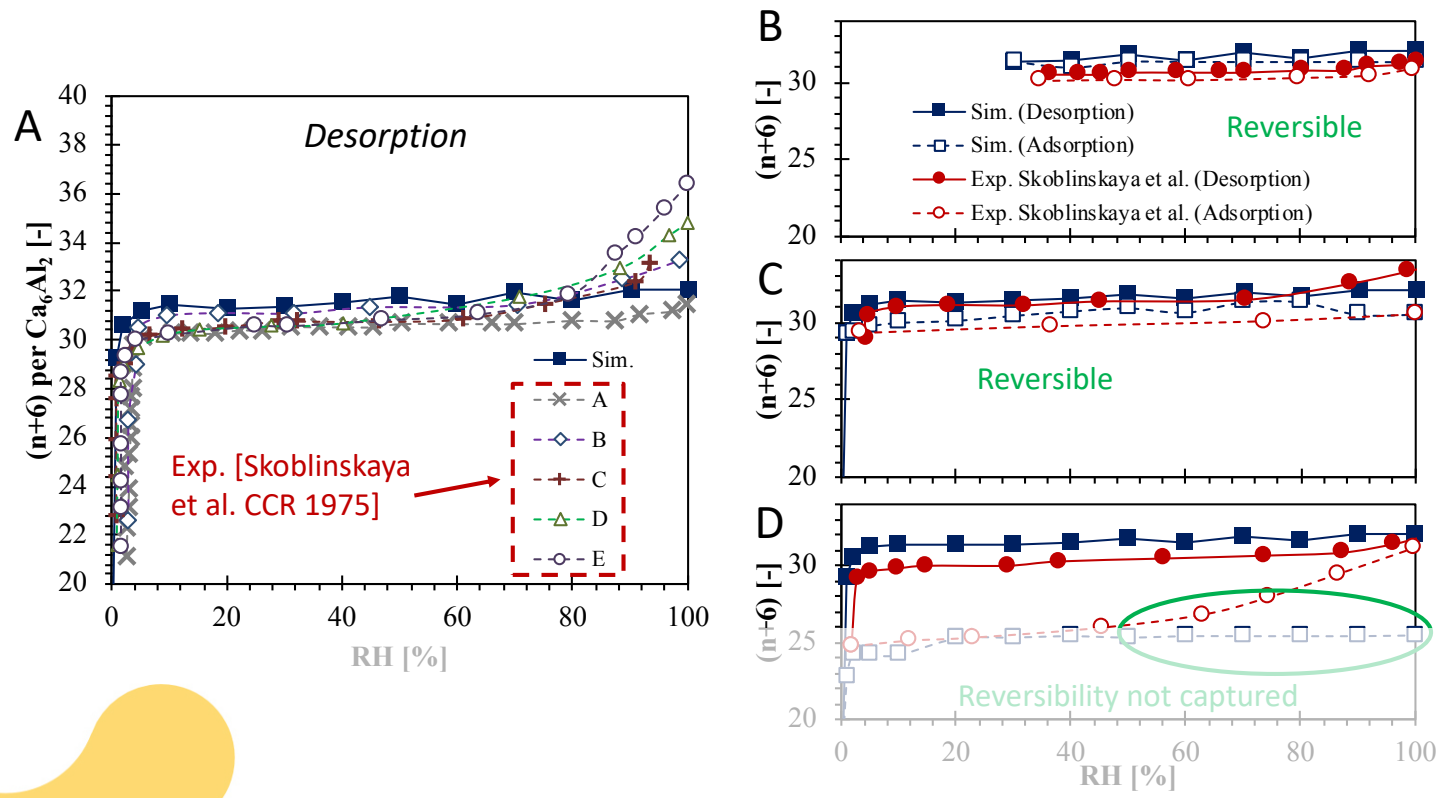

Figure 1: Sorption isotherms obtained from simulation compared against Skoblinskaya et al. [5] experimental data $(\mathrm{T}=291 \mathrm{~K})$. (A) Desorption branch compared to the experimental sets A-E from [5]. (B) and (C) Sorption cycles with close loop reversible hysteresis from 3à and 1 \% RH, respectively. (D) Sorption cycles with open loop hysteresis: simulation cannot capture the reversibility under adsorption.

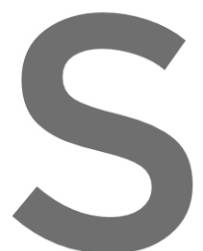

the (symmetric) stress tensor are controlled independentiy. Note that

of ettringite, the strategy

(as generally adopted in in

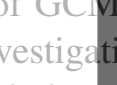

C-NVT simulatio

ns having
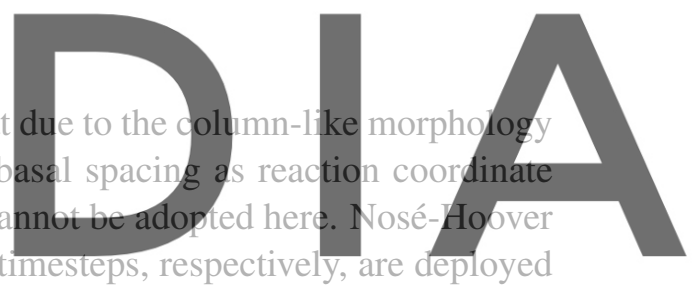

in the NoT stages

Register for free at https//www.scipedia.com to download the version without the watermark

\section{RESULTS AND DISCUSSION}

\subsection{Sorption Isotherms}

Figure 1 shows the sorption isotherms obtained from simulations compared against the experimental results of Skoblinskaya et al. [5]. The desorption branch obtained by simulation is in agreement with the experimental data. In Figures 1(B)-(D) sorption cycles (desorption and adsorption branches) are shonw from three reweting RH as in ref. [5]. For the cycles with rewetting from 30 (B) and $1 \%$ (C) of RH, close loop reversible hysteresis is observed by both experiments and simulations. For the cycles with rewetting at $0.1 \% \mathrm{RH}$, the reversibility of the adsorption branch at high $\mathrm{RH}$ is not captured by the simulations.

Direct simulations in the osmotic ensemble, as proposed in this study, have been successfully used to model sorption and hysteresis in phases with small volume change [7]. However, the literature on microporous phases in which sorption is associated with large volume changes (see, for instance, Bousquet et al. [16] for MOFs) shows that direct simulations in the osmotic ensemble may not capture the reversibility. Other better-suited simulation techniques (e.g. Wang-Landau free energy) should be adopted 
to model this specific phenomenon. Figure 2 shows that the conversion of ettringite into metaettringite is associated with large changes in the lattice parameters (in agreement with the experimental evidence from various authors). Therefore, it is expected that the reversibility in the adsorption cycle is not observed in the simulations at high RH as is the case in the experiments.
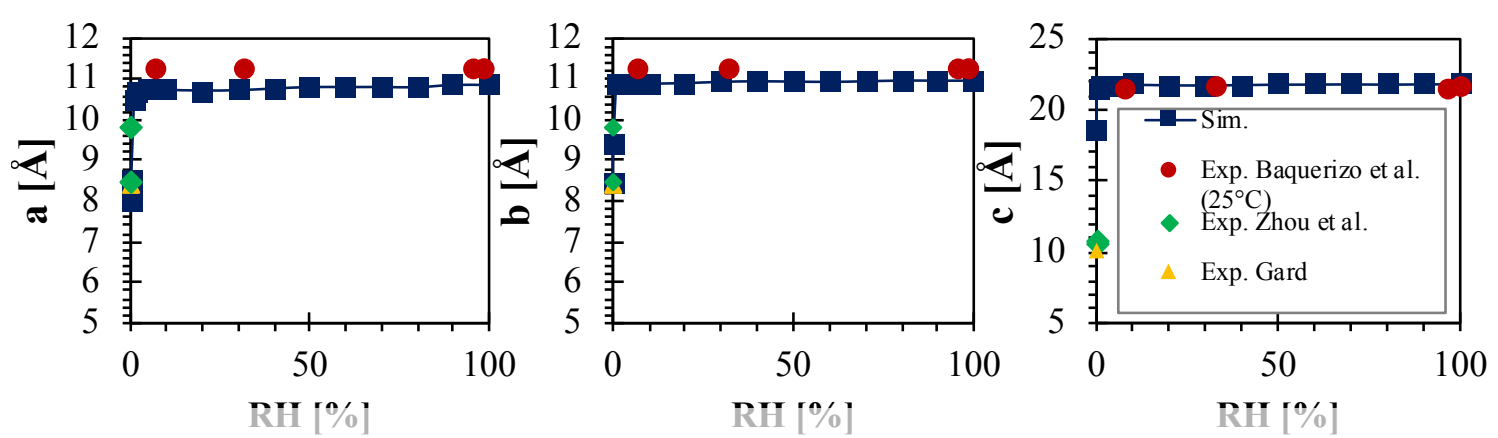

Figure 2: Anisotropic volume changes under desorption: comparison of lattice parameters changes obtained from simulations against Baquerizo et al. [3] experimental data ( $\mathrm{T}=298 \mathrm{~K})$, Zhou et al. [4], and Gard [15].

3.2

The structure of metaettringite

The good agreement of simulation with the expertimental data on the desorption isotherm and lattice
parameters changes under desorption buids confidence in using dur simulation approach to analyze
the structural changes associated with metaettringite formation. Figure 3 (A) shows a comparison of
experimental and simulated X-ray diffraction patterns of ettringite at various RH and metaetringite. For
comparison, the experimental pattern of depujolsite, a mineral associated with metaettringite [4]. is also
shown. Experimental and simulated patterns in the ettringite domain are consistent. Most of the peaks in the ettringite pattern disappear in the metaettringite patterns ( $\mathrm{RH}$ of 0.1 and $0 \%$ in the simulated

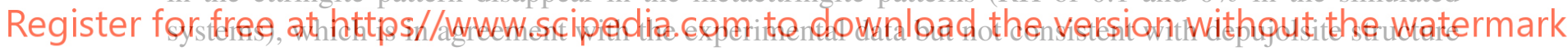

as suggested by Zhou et al. [4]. Figure 3 (B) selected snapshots of simulated systems shows how the

columns, which are very well defined in ettringite, are not well defined in metaettingite structures. This observation is in agreement with experimental XRD patterns from Baquerizo et al. [3].

The lack of well-defined peaks in metaettringite XRD patterns and the visual analysis of the snapshots in Figure 3 (B) indicate that metaettringite is a more disordered phase. To quantify the relative order in a molecular system, we use the concept of excess entropy. The pair (first-order) excess entropy $S_{2}$ can be computed from the pair radial distribution functions $g_{i-j}$ using [17]:

$$
\frac{S_{2}}{k N}=-\frac{2 \pi}{V} \sum_{i, j} \int_{0}^{\infty}\left[g_{i-j}(r) \ln g_{i-j}(r)-g_{i-j}+1\right] r^{2} d r
$$

where $N$ is the total number of particles and $x_{i}$ is the molecular fraction of particle of type $i$ in the system. Figure 4 shows the total excess entropy $S_{2}$ in the desorption branch. The excess entropy is significantly higher in metaettringite when compared to ettringite. 


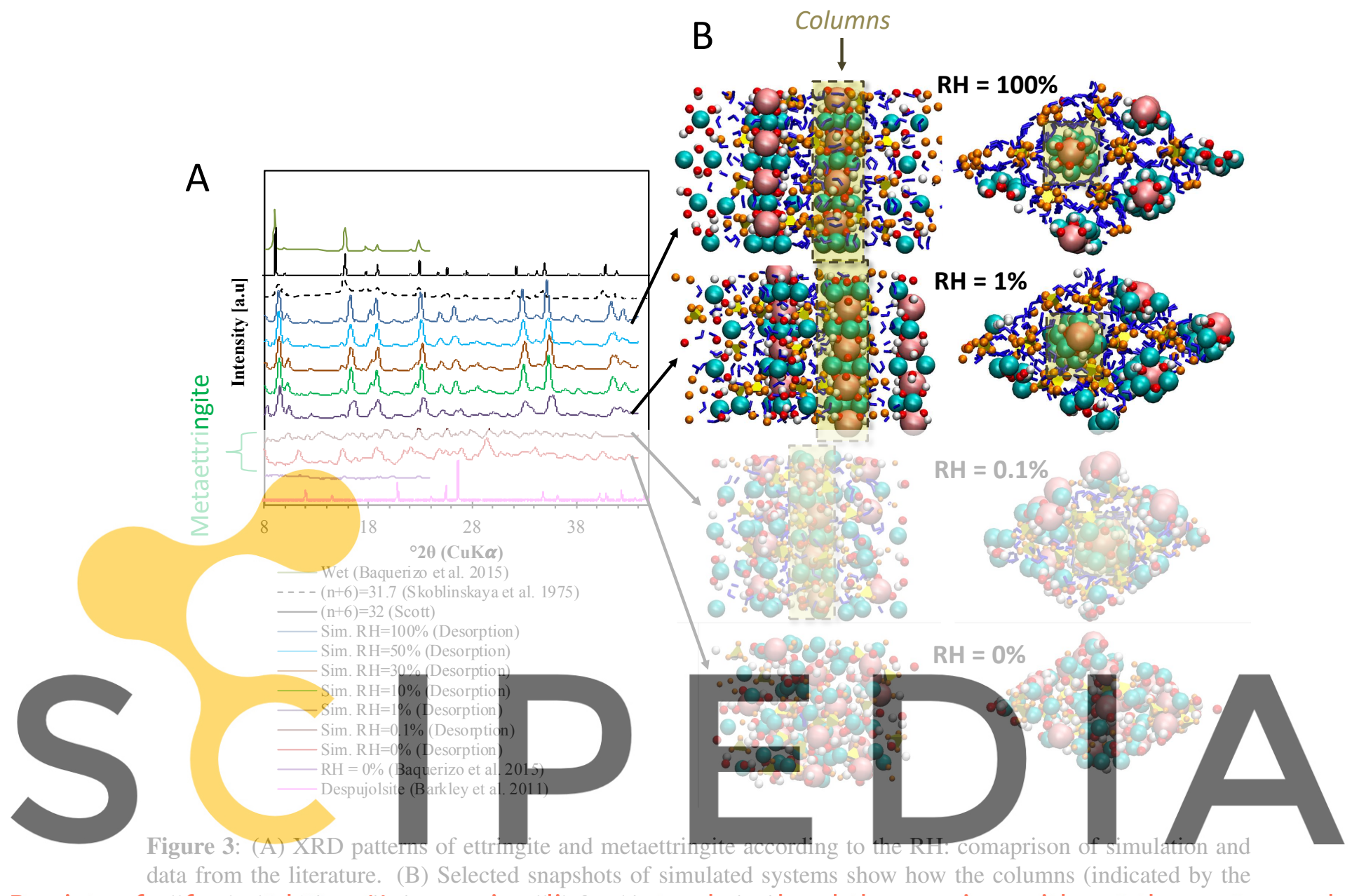

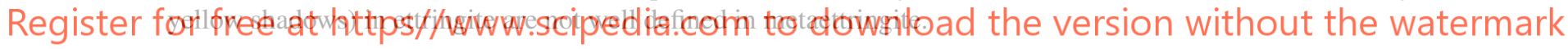

\section{CONCLUSIONS}

In this work, we performed molecular simulations to get a fundamental understanding of the origins of sorption behavior of ettringite from the relevant scale of the adsorption phenomenon (i.e. the molecular scale). The simulations capture the water content and lattice parameters changes in ettringite under desorption, which allows investigating the structural changes that lead to metaettringite formation. We analyze, for the first time, the structural features of metaettringite with atomic-level detail.

The results presented here provide fundamental understanding of sorption processes in ettringite that may contribute to new strategies to optimize and conceive new AF-phases rich materials for tailored applications (e.g. thermal energy storage) as well as shed light on the role of ettringite in drying shrinkage of cement-based materials [18]. Other perspectives of this work include further developments to properly capture reversibility in metaettringite-to-ettringite conversion under adsorption and the effects of the temperature under drained and undrained conditions [19]. The analysis of the role of H-bonds role in sorption may provide interesting physical insights to understand the origins of hysteresis in ettringite 


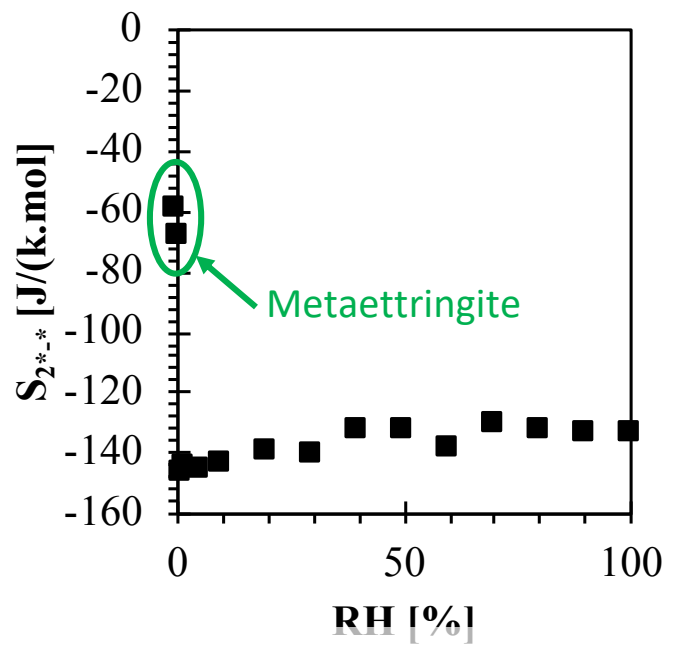

Figure 4: Entropy as function of the RH in the desorption branch.

sorption.

REFERENCES

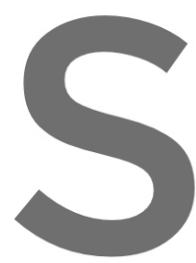

REFERENCES

[1] Chen B, Kuznik P,

thermal energy stora

[2] Struble LJ, Brown P

storage. Report (1984)

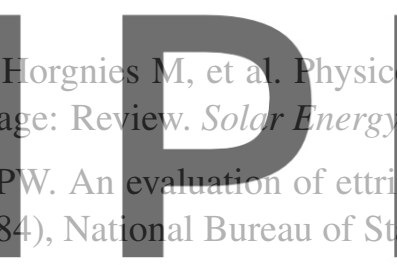

Ochemical properties of ettringite/meta-e
Materiats and Solar Cells (2019) 193:3
ingite and related compounds for use in
andards, Gaithersburg, MD

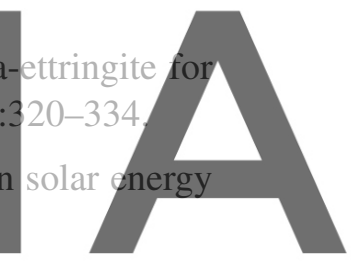

[3] Baquerizo LG, Matschei T, Scrivener KL. Impact of water activity on the stability of ettringite.

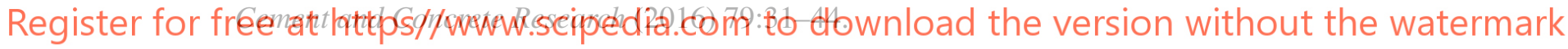

[4] Zhou Q, Lachowski EE, Glasser FP. Metaettringite, a decomposition product of ettringite. Cement and Concrete Research (2020) 34:703-710.

[5] Skoblinskaya NN, Krasilnikov KG, Nikitina LV, Varlamov VP. Changes in crystal structure of ettringite on dehydration. Cement and Concrete Research (1975) 5:419-431.

[6] Honorio T, Guerra P, Bourdot A Molecular simulation of the structure and elastic properties of ettringite and monosulfoaluminate. Cement and Concrete Research (2020) 135:106126.

[7] Honorio T, Chemgne Tamouya OM, Shi Z, Bourdot A. Intermolecular interactions of nanocrystalline alkali-silica reaction products under sorption. Cement and Concrete Research (2020) 136:106155.

[8] Honorio T. Monte Carlo Molecular Modeling of Temperature and Pressure Effects on the Interactions between Crystalline Calcium Silicate Hydrate Layers. Langmuir (2019) 35:3907-3916.

[9] Honorio T., Brochard L., Vandamme M. Hydration Phase Diagram of Clay Particles from Molecular Simulations. Langmuir (2017) 33:12766-12776. 
[10] Moore AE, Taylor HFW. Crystal structure of ettringite. Acta Crystallogr Sect B (1970) 26:386-393.

[11] Cygan RT, Liang J-J, Kalinichev AG. Molecular Models of Hydroxide, Oxyhydroxide, and Clay Phases and the Development of a General Force Field. J Phys Chem B (2004) 108:1255-1266.

[12] Cannon WR, Pettitt BM, McCammon JA. Sulfate Anion in Water: Model Structural, Thermodynamic, and Dynamic Properties. J Phys Chem (1994) 98:6225-6230.

[13] Liu L, Jaramillo-Botero A, Goddard WA, Sun H. Development of a ReaxFF Reactive Force Field for Ettringite and Study of its Mechanical Failure Modes from Reactive Dynamics Simulations. $J$ Phys Chem A (2012) 116:3918-3925.

[14] Berendsen, H. J. C., Grigera, J. R., Straatsma, T. P. The missing term in effective pair potentials. $J$ Phys Chem (1987) 91(24):6269-6271.

[15] Gard, J.A. in: H.F.W. Taylor (Ed.), Chemistry of Cements, vol. 2, Academic Press, London (1962) pp. $243-270$. Chapter 24.

[16] Bousquet D., Coudert F-X., Boutin A. Free energy landscapes for the thermodynamic understanding of adsorption-induced deformations and structural transitions in porous materials. J Chem Phys (2012) 137:044118.

[17] Baranyai, A., Evans, D. J. Direct entropy calculation from computer simulation of liquids. Physical Review A (1989) 40(7): 3817-3822.

[18] Qomi M. J. A., Brochard L., Honorio T., Maruyama I., Vandamme M. Advances in Atomistic

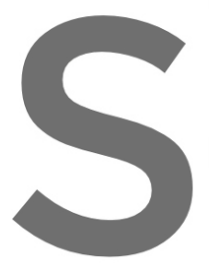
Modeling and Understanding of Drying Shrinkage in Cemen Research (2021)

19] Brochard L., Honorio T . without assuming Gibbs-Duhem equation. International Journal 152:103296.
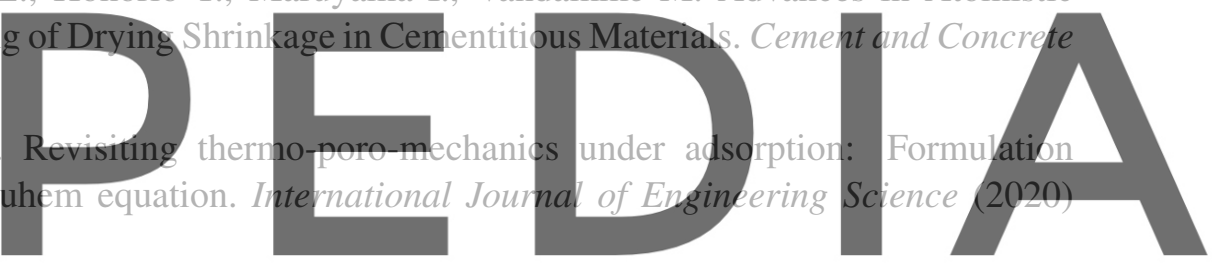